\title{
Discrete Set of Separations between Phase-Locked Soliton Pairs in a Passively Mode-Locked Fiber Laser
}

\author{
Ph. Grelu, F. Belhache, Laboratoire de Physique de l'Université de Bourgogne, UMR 5027, B.P. 47870, 21078 Dijon, \\ France \\ J. M. Soto-Crespo, Instituto de Optica, Consejo Superior de Investigaciones Científicas, Serrano 121, 28006 Madrid, \\ Spain \\ N. N. Akhmediev, Optical Science Centre, The Australian National University, Canberra, ACT 0200, Australia
}

Passively mode-locked fiber ring lasers and dispersion-managed fiber transmission links are examples of optical dissipative dynamical systems, supporting stable pulses and multi-soliton trains. An example of stable multi-soliton solution with $+/-\square / 2$ phase differences, have been predicted theoretically earlier based on the model of complex quintic Ginzburg-Landau equation [1]. More recently, we demonstrated experimentally the existence of such stable $+/-\square / 2$ phase-locked soliton pairs in a passively mode-locked fiber ring laser [2]. The ring laser consists of a 1.6-meter length of single-mode erbium-doped fiber, an optical isolator for unidirectional lasing, a 4.8-meter length of standard telecom fiber, and is pumped by $980 \mathrm{~nm}$ laser diodes. Pumping power ranges from 40 to $300 \mathrm{~mW}$. Passive mode locking is achieved through the nonlinear polarization evolution in the slightly birefringent fibers, followed by intensity discrimination of the polarization states with a passive polarizer. We use the same system in the present work. The phase-locked soliton pairs generated consist of two pulses, whose individual duration is around 500 fs, separated by a fixed time interval. Depending on the mode-locking conditions and other parameters, this time interval ranges from 5 to 40 pulse widths.

In this present work, we show experimentally and numerically that for a given laser configuration, only a discrete set of separations is accessible. The model used in the numerical simulations is similar to our experimental setup. It is based on parameter management technique with periodic pulse propagation in various parts of the cavity, and includes nonlinearity, dispersion, birefringence, gain saturation and spectral filtering. This model reproduces well the formation of stable soliton pairs. In the figures below, we plot the time separation of the pulse pair $\square$ along the horizontal axis, while the relative phase is plotted along the vertical axis. Each time unit equals to $150 \mathrm{fs}$ in the experiment. Fig. 1(a) clearly demonstrates the formation of a stable phase-locked soliton pair with fixed separation and fixed phase difference, from an initial condition of two pulses with an arbitrary separation and an arbitrary phase difference within certain limits. When starting from initial separations and phase differences beyond those limits, the pulse pair evolves towards phase-locked states having discrete possible values for their separation (see Fig. 1b). These separations are regularly spaced. Our experimental results confirm the existence of these quantized separations of the phase-locked soliton pairs.

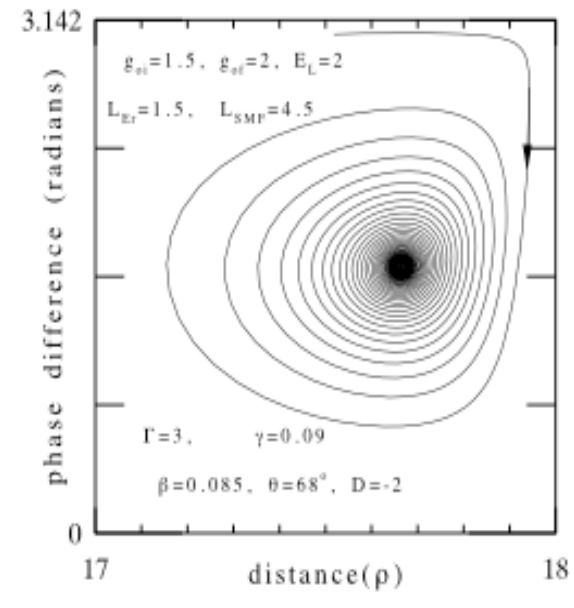

Fig. 1(a)

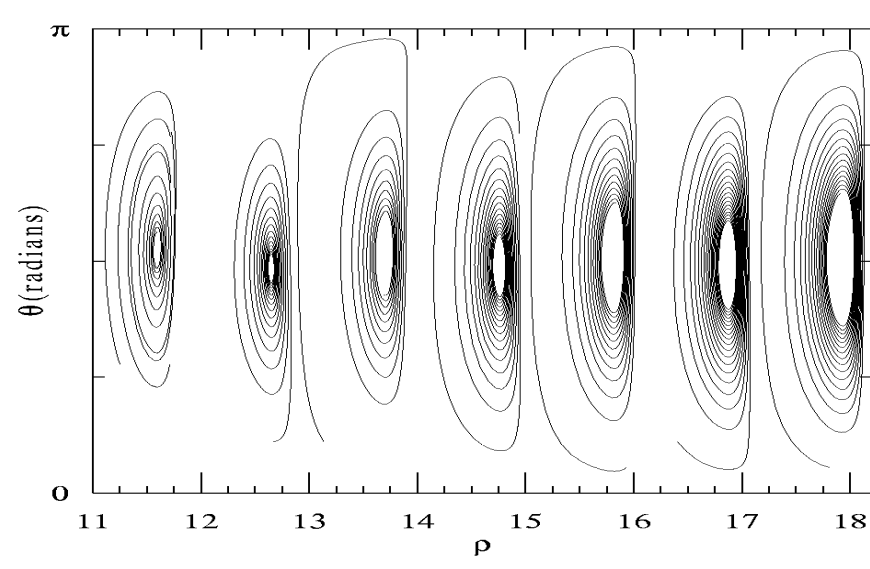

Fig. 1(b)

[1] N. N. Akhmediev, A. Ankiewicz, and J. M. Soto-Crespo, "Stable soliton pairs in optical transmission lines and fiber lasers", J. Opt. Soc. Am. B 15, 515 (1998).

[2] Ph. Grelu, F. Belhache, F. Gutty, and J. M. Soto-Crespo, "Phase-locked soliton pairs in a stretched-pulse fiber laser", Opt. Lett. 27, 966-968 (2002). 